\title{
APROXIMACIONES BIOÉTICAS AL CASO DE CHARLIE GARD
}

\author{
Horacio L. Salgado-Fernández ${ }^{1}$
}

Resumen: El litigio que enfrentó a los padres del niño Charlie Gard contra el Great Ormond Street Hospital de Londres, primero, y contra el propio Reino Unido, después, generó efectos mediáticos a nivel mundial pero escaso debate desde la perspectiva bioética. En el presente artículo se resumen algunos aspectos relevantes de la controversia que permiten analizar tres de las lecciones formuladas por Wilkinson y Savulescu al respecto. Se plantean además dos reflexiones bioéticas: la primera en relación a la dificultad en la aplicación de principios para examinar el caso, y la segunda referida a la interdisciplinariedad requerida para su análisis.

Palabras clave: bioética, filosofía, problemas bioéticos, ética basada en principios, análisis ético

\section{Bioethical approximations to the case of Charlie Gard}

Abstract: The litigation that confronted the parents of the child Charlie Gard against the Great Ormond Street Hospital in London, first, and against the United Kingdom itself, later, generated media effects worldwide but little debate from a bioethical perspective. This article summarizes some relevant aspects of the controversy that allow analyzing three of the lessons formulated by Wilkinson and Savulescu in this regard. Two bioethical reflections are also proposed: the first in relation to the difficulty in the application of principles to examine the case, and the second referred to the interdisciplinarity required for its analysis.

Keywords: bioethics, philosophy, bioethics issues, principle-based ethics, ethical analysis

\section{Aproximaçóes bioéticas ao caso de Charlie Gard}

Resumo: O litigio que enfrentou os pais do menino Charlie Gard contra o Great Ormond Street Hospital de Londres, primeiro contra o próprio Reino Unido, gerou depois efeitos midiáticos mundiais porém escasso debate sob uma perspectiva bioética. No presente artigo se resume alguns aspectos relevantes da controvérsia, que permitem analisar três das liçóes formuladas por Wilkinson e Savulescu a respeito. Além disto, se propõe duas reflexôes bioéticas: a primeira em relação à dificuldade na aplicaçáo de princípios para examinar o caso e a segunda no que se refere à interdisciplinaridade requerida para sua análise.

Palavras chave: bioética, filosofia, problemas bioéticos, ética baseada em princípios, análise ético

\footnotetext{
${ }^{1}$ Facultad de Psicología, Universidad San Sebastián, Chile. Comité Ético Científico, Universidad San Sebastian, Chile.

Correspondencia: horacio.salgado@uss.cl
} 


\section{Introducción}

El doloroso caso del niño londinense Charlie Gard acaparó durante 2017 la atención de los medios de prensa de todo el mundo; sin embargo, estuvo lejos de gatillar un debate público desde un punto de vista ético(1) o bioético.

El propósito del presente artículo es discutir tres asuntos bioéticos que resultan de especial interés en relación al caso, junto con formular dos reflexiones de interés general. Para lograr lo señalado se sintetizan a continuación algunos hechos relevantes del caso.

Charlie Gard nació el 4 de agosto de 2016 aparentemente sano(2). Sus padres, Chris Gard y Constance Yates, advirtieron a las pocas semanas que Charlie presentaba debilidad muscular y el 11 de octubre fue ingresado al Great Ormond Street Hospital donde se le diagnosticó "síndrome de depleción mielodisplásica del ADN mitocondrial de aparición infantil", denominado generalmente MDDS"(3). La enfermedad había provocado diversos efectos: el cerebro, músculos y capacidad para respirar de Charlie estaban severamente afectados; padecía sordera congénita y un trastorno de epilepsia grave; su corazón, hígado y riñones también estaban afectados; la debilidad muscular progresiva le impedía mover sus extremidades o respirar sin ayuda(2,3); adicionalmente, la rápida evolución del trastorno tenía como pronóstico una muerte dentro de un breve periodo de tiempo(3).

A principios de 2017, el equipo médico planificó un tratamiento experimental con nucleósidos, que había sido utilizado por el Dr. Hirano en el Columbia University Medical Center para una forma distinta del síndrome $(1,2)$. Existía acuerdo en que el tratamiento podría tener alguna posibilidad de beneficio solamente en el caso de que no hubiese daño cerebral irreversible(4). Se programó para el 13 de enero una solicitud de respuesta rápida al Comité de Ética del hospital londinense para poder proceder al tratamiento $(1,4)$. Desafortunadamente, Charlie empezó a tener convulsiones poco antes de Navidad(4), las que aproximadamente entre el 9 y el 27 de enero se incremen$\operatorname{taron}(1)$, siendo el tratamiento desechado como alternativa debido a evidencia de daño cerebral irreversible, lo que condujo al equipo médico a concluir que tal terapia, así como los cuidados intensivos, resultarían inútiles(4). Todos los médicos tratantes estuvieron de acuerdo en que se debía retirar la ventilación artificial y brindar solo cuidados paliativos a Charlie, permitiéndole morir en paz y con dignidad(3).

Los padres reunieron entonces el dinero mediante una campańa para realizar el tratamiento directamente en Estados Unidos, para lo cual debían trasladar a Charlie. Ante tal posibilidad, a fines de febrero, el equipo médico del Hospital presentó un requerimiento a la Corte de Justicia para poder desconectar a Charlie de los soportes vitales y proceder a cuidados paliativos, con el consiguiente rechazo de los padres(1). Tras varias audiencias, el juez Nicholas Francis terminó fallando a favor del Hospital(3). A fines de mayo, la decisión fue confirmada por la Corte de Apelaciones(5) y, en junio, por la Corte Suprema(6). Luego, la Corte Europea de Derechos Humanos ratificó la sentencia de los tribunales británicos(1). El dictamen del juez Francis consideró la evidencia médica y las opiniones de expertos, aun cuando declaró que, en cualquier caso, lo que se buscaba era el mejor interés de Charlie desde el punto de vista legal(3). Un aspecto relevante de la evidencia que el propio juez citó textualmente fue la consideración del médico estadounidense, tras hablar con el equipo médico del Great Ormond Street Hospital, en relación a que "ver los documentos esta mañana ha sido muy útil. Puedo entender las opiniones de que está tan severamente afectado por la encefalopatía que cualquier intento de terapia sería inútil. Estoy de acuerdo en que es muy poco probable que mejore con esa terapia. Es improbable"(3:5).

Pese a ello, el Dr. Hirano posteriormente indicó que presentaría nueva evidencia de posibles beneficios del tratamiento experimental, lo que animó a los padres y al propio equipo médico del Great Ormond Street Hospital(4). No obstante, posteriormente el equipo médico señaló estar sorprendido y decepcionado, ya que quedó de manifiesto que el Dr. Hirano no solo nunca había visitado a Charlie en el Hospital sino que tampoco había leído su historial médico, revisado sus imágenes cerebrales o leído las segundas opiniones expertas de la condición del niño; por lo demás, quedó expuesto un posible interés financiero en algunos 
compuestos que el Dr. Hirano proponía prescribir a Charlie(4).

Finalmente, con el acuerdo de sus padres, el 27 de julio Charlie Gard fue desconectado de los soportes vitales falleciendo al día siguiente, pocos días antes de alcanzar el primer año de vida(7).

Concluido este resumen, es posible señalar que son escasas las reflexiones éticas o bioéticas en torno al caso que han sido desarrolladas a la fecha. Una de ellas es la elaborada por Wilkinson y Savulescu, quienes sintetizan nueve lecciones que se pueden derivar del caso de Charlie(8). Me centraré en analizar tres de tales lecciones y, posteriormente, desarrollaré dos planteamientos no considerados por dichos filósofos, pero que considero pertinentes y de interés bioético.

\section{Rol de los padres respecto de las decisiones so- bre sus hijos}

La primera lección de Wilkinson y Savulescu a la que me referiré consiste en el rol que les concierne a los padres en la toma de decisiones sobre sus hijos. Para los filósofos, lo primordial es la definición de lo que constituye "un nivel suficiente - $\mathrm{O}$ posibilidad- de daño para justificar o no una determinada acción médica” (8). En ese contexto, los filósofos recuerdan que el mismo equipo médico había declarado que el daño neurológico era tan grave que Charlie estaba "más allá de la experiencia” y, por lo tanto, resultaría lícito preguntarse en tales circunstancias si aceptar la solicitud de tratamiento de los padres hubiese constituido, así las cosas, un daño realmente significativo.

El caso de Charlie Gard evidencia, de este modo, una perspectiva diametralmente distinta a diversos análisis de situaciones en que la voluntad de los responsables de un paciente llega a generar conflicto con las indicaciones de un equipo médico(9). Casos como los de Baby Doe, Robinson Gómez, Daniel Hause, Kara y un niño chileno de 11 años, en que los padres rechazaron tratamiento para sus hijos y los equipos médicos en varios casos lucharon en la Corte para poder proporcionar tales tratamientos(9). En el caso de Charlie Gard la situación fue totalmente distinta: los padres fueron quienes lucharon para efectuar una terapia experimental y el equipo médico batalló por evi- tarla (cada cual con sus argumentos y evidencias) (10).

Una de las presunciones occidentales en relación a la autoridad de los padres - la referente a que, debido a los naturales lazos de afecto, los padres actúan siempre buscando el mejor interés de los hijos- es refutable, debido a lo complejo que resulta definir el "mejor interés del niño"(9); en el caso de los padres que objetan procedimientos que podrían salvar la vida de los niños, ya sea por convicciones religiosas o debido a malas experiencias, es fácil evidenciar esta refutación. En cualquier caso, el fondo de la discusión en esta presunción es la definición y esclarecimiento de lo que es "el mejor interés para el niño"; en esto, de hecho, se centró explícitamente el fallo del juez Nicholas Francis. Pero, ¿cuáles son los criterios que posibilitan determinar el mejor interés para un niño? La decisión sobre el mejor interés depende de una opinión objetiva sobre los fenómenos subjetivos del dolor y del sufrimiento(7), donde cabe la argumentación de que podrían haberse aplicado medidas analgésicas y sedativas de tipo estándar en caso de que hubiese dudas sobre alguna posibilidad de dolor en Charlie. Asimismo, se sostiene que la expresión "el mejor interés" podría no ser sino un eufemismo, especialmente si existe un espacio para desacuerdo razonable, por ejemplo, una posibilidad aunque sea ínfima de mejora(11).

Adicionalmente, esta decisión está cruzada por el principio de autonomía(12), en este caso de los padres sobre su hijo y de la posibilidad de límites a dicha autonomía. El caso de Charlie desafía a pensar en los límites de los derechos de los padres, así como a elaborar principios que posibiliten resolver si los padres deben decidir en situaciones en que: existen dudas sobre el dolor en un paciente, un médico está dispuesto a administrar un tratamiento seguro que podría ser beneficioso, los padres quieren probar ese tratamiento, y la única alternativa a la terapia experimental es la muerte(13).

\section{Recursos económicos y principio de justicia}

Otra de las lecciones formuladas por Wilkinson y Savulescu es la referente a los recursos económicos, considerando que un tratamiento demasiado 
oneroso no debiera ser aplicado si eso merma la posibilidad de que otras personas obtengan beneficios de salud(8). Si bien en el caso de Charlie los padres habían conseguido los recursos por su cuenta, mediante una campaña de recolección de fondos, los filósofos recuerdan la afirmación de Truog de que la preocupación por los recursos finitos de los sistemas de salud es legítima incluso si los padres o aseguradores son capaces de costear el tratamiento, puesto que la posibilidad de que existan centros terciarios de salud que realicen investigación depende de los recursos de una sociedad o comunidad determinada, y en ese sentido es legítima la exigencia de que tales recursos se usen sabiamente( 7$)$.

A pesar de lo anterior, podrían usarse los mismos argumentos económicos para apoyar el tratamiento de Charlie al otro lado del Atlántico (en Estados Unidos), considerando que se podría haber desocupado una unidad de cuidados intensivos que muy probablemente otros niños requerían de modo vital(8). Por cierto, tras estas discusiones es posible reconocer el principio de justicia planteado por Beauchamp y Childress, según el cual se requiere hacer un uso racional de los recursos sanitarios, considerando un análisis de costobeneficio adecuado, pues un manejo muy costoso sería antiético(12).

\section{Dignidad y proporcionalidad terapéutica}

Finalmente, una tercera lección que formulan Wilkinson y Savulescu se refiere a la necesidad de un mayor análisis ético, especialmente respecto de cuestiones normativas: los filósofos se refieren particularmente al concepto de "dignidad" utilizado por el juez Nicholas Francis como argumento en su sentencia(8). Este planteamiento está en sintonía con la argumentación de que, a pesar de su aparición permanente en discusiones (por ejemplo, sobre la eutanasia), el concepto de dignidad no ofrece criterios claros para tomar decisiones (14).

Es lícito "dejar morir" cuando la prolongación artificial de la vida implica excesiva carga para el paciente y su familia, y cuando la medicina no ofrece resultados(14). Se hace necesario decidir cuándo el soporte artificial se torna inútil y cuándo se debe permitir que la muerte sobrevenga(15). Si las terapias solo prolongan la agonía y producen sufrimiento al paciente y a su familia, dejar morir pasa a ser un imperativo ético(14). En cualquier caso, es sumamente complejo determinar cuándo es correcto dejar morir y cuándo no(14), lo que queda particularmente claro en el caso de Charlie Gard. Posiblemente, para el equipo médico del Great Ormond Street Hospital, someter a Charlie al tratamiento experimental significaba una suerte de "encarnizamiento terapéutico".

Por lo anterior, Wilkinson y Savulescu afirman que se hace sumamente necesaria una descripción objetiva de la frase "una vida digna de ser vivida", que sea lo suficientemente sólida y clara como para ser aplicada a diversidad de casos, respetando los razonables desacuerdos sobre valor y valores(8).

Por otro lado, está la argumentación en relación al principio de proporcionalidad terapéutica, de acuerdo al cual no se está obligado sino a utilizar aquellas medidas terapéuticas que ofrezcan una razonable probabilidad de beneficio(16). Este principio distingue entre medidas ordinarias y extraordinarias. En el caso del niño Charlie Gard las medidas que se discuten son extraordinarias o desproporcionadas en relación al posible beneficio y no se está moralmente obligado a utilizarlas $(16,17)$. Dos de los elementos a considerar en esta evaluación de la proporcionalidad y que estarían en contra del uso del tratamiento experimental en el caso de Charlie, son la utilidad de la medida terapéutica y los costos que podría conllevar.

Ahora bien, el que no se esté moralmente obligado a efectuar un tratamiento no significa que no se pueda realizar; en esa línea, si los médicos hubieran aplicado la terapia de nucleósidos en el mismo momento que iniciaron la batalla legal, podría haberse sabido al menos si el tratamiento funcionaba incluso antes de que el juicio llegara a su término(9). Como dice Lantos(13:936) "seguramente ese hubiese sido un mejor resultado para todos los involucrados". Cobra sentido, además, la petición de bajar los umbrales para los tratamientos experimentales y permitirlos más tempranamente en casos cuya única alternativa es la muerte(8). 


\section{Discusión}

Quisiera, a continuación, desarrollar dos reflexiones, no formuladas por Wilkinson y Savulescu, que me parecen relevantes. La primera, que me ha permitido delinear algunos aspectos del análisis de las lecciones anteriores, es la siguiente: el caso del niño Charlie Gard ha generado tanto interés en los medios de comunicación debido a que en él es posible identificar, en mayor o menor medida, y en especial tensión, cada uno de los principios planteados por Beauchamp y Childress para el análisis de los problemas bioéticos en el campo de la biomedicina: beneficencia, no maleficencia, autonomía y justicia.

En ese contexto, sugiero que el interés del público brota de la especial dificultad para discernir qué aplicabilidad tienen estos principios en el caso. Así, por ejemplo, el principio de beneficencia es probablemente lo que impulsó al equipo médico a solicitar la desconexión de los soportes vitales y aplicar cuidados paliativos, a fin de dejar morir y evitar el sufrimiento de Charlie; pero, del mismo modo, se podría argüir que los padres de Charlie buscaban la beneficencia de su hijo cuando intentaban obtener un tratamiento experimental que pudiera prolongar su vida y, quizás, aunque muy poco probablemente, mejorar la calidad de ésta.

Por otra parte, el principio de no maleficencia podría esgrimirlo el equipo médico en el sentido de no dañar a Charlie, esto es, no generarle sufrimiento o estrés innecesario al permitir un potencial viaje y tratamiento, cuyas probabilidades de éxito eran probablemente nulas. $\mathrm{Al}$ contrario, el principio de no maleficencia podría fundar una argumentación por parte de los padres, relativa a que dejar morir a su hijo sin realizar un tratamiento experimental, por muy costoso que fuera, no sería otra cosa que dañarlo.
Los principios de justicia y de autonomía ya fueron expuestos en párrafos previos, en relación al uso adecuado de los recursos económicos y sanitarios, y a los límites en cuanto a las decisiones de los padres sobre sus hijos, respectivamente.

Vinculado con lo anterior, sería esclarecedor realizar un análisis del caso desde algún modelo de análisis de problema ético clínico, con el fin de dilucidar una adecuada aplicación de los principios mencionados.

Finalmente, una segunda reflexión es la necesidad de una perspectiva interdisciplinaria de estas situaciones particularmente complejas. El hecho de que este tema haya sido tratado desde un punto de vista médico, legal, espiritual, filosófico, evidencia el desafío de generar espacios de discusión argumentada, que permitan superar las dificultades que los diversos autores han esbozado tanto para este caso como en relación a otros.

Los problemas bioéticos requieren una mayor articulación y comprensión entre las distintas disciplinas involucradas. Ciertamente, este no es un desafío simple, pero no es posible que una sola disciplina — cualquiera que esta sea - se arrogue el derecho de asumir una última palabra en torno a alguna controversia. En el caso del niño Gard es posible apreciar el esfuerzo que cada una de las disciplinas hizo para aportar en la resolución de una dolorosa situación humana. En ese sentido, la disposición a poner el propio conocimiento al servicio del debate racional resulta tremendamente propicia en un escenario - la bioética- en el que los hechos y los valores suelen convivir en constante tensión. 


\section{Referencias}

1. Savulescu J, Singer P. Unpicking what we mean by best interests in light of Charlie Gard. The BMJ Opinion (Internet). 2017 Aug 2. Disponible en: http://blogs.bmj.com/bmj/2017/08/02/unpicking-what-we-mean-by-best-interests-inlight-of-charlie-gard/

2. European Court of Human Rights. First Section Decision. Application no. 39793/17 Charles GARD and Others against the United Kingdom (Internet), 2017. Disponible en https://hudoc.echr.coe.int/eng\#\{“itemid”:[“001-175359”]\}

3. Francis N. Neutral Citation Number: [2017] EWHC 972 (Fam). Case No. FD17P00103. In The High Court of Justice Family Division (Internet), 2017. Disponible en https://www.judiciary.gov.uk/wp-content/uploads/2017/05/gosh-vyates-and-gard-20170411-1.pdf

4. Great Ormond Street Hospital. Great Ormond Street Hospital Position Statement at High Court on 24 July 2017 (Internet) 2017. Disponible en: http://www.gosh.nhs.uk/news/latest-press-releases/gosh-position-statement-issued-highcourt-24-july-2017

5. McFarlane A. Neutral Citation Number: [2017] EWCA Civ 410. Case No: B4/2017/1236/PTA+A. In The Court of Appeal (Civil Division) on Appeal from The High Court of Justice (Family Division) in The Matter of Charles Gard (DOB 4/8/16) (Internet), 2017. Disponible en: http://www.bailii.org/ew/cases/EWCA/Civ/2017/410.html

6. Judgment of the UK Supreme Court in The Case of Charlie Gard (Internet), 2017. Disponible en https://www.supremecourt.uk/cases/docs/charlie-gard-190617.pdf

7. Truog RD. The United Kingdom Sets Limits on Experimental Treatments. The Case of Charlie Gard. JAMA 2017; 318(11): 1001-1002.

8. Wilkinson D, Savulescu W. Hard lessons: learning from the Charlie Gard case. J Med Ethics 2017; 0: 1-5. doi:10.1136/ medethics-2017-104492

9. McNab ME, Beca JP. ¿Existen límites en la decisión de los padres sobre el tratamiento de sus hijos? Rev Chil Pediatr 2010; 81(6): 536-540.

10. Great Ormond Street Hospital. Great Ormond Street Hospital Position Statement 13 July 2017. (Internet) 2017. Disponible en http://www.gosh.nhs.uk/news/latest-press-releases/latest-statement-charlie-gard

11. Savulescu J. The moral of the case of Charlie Gard: give dying patients experimental treatment... early. (Internet) 2017. Disponible en http://blog.practicalethics.ox.ac.uk/2017/07/the-moral-of-the-case-of-charlie-gard-give-dying-patientsexperimental-treatment-early/

12. Beauchamp T, Childress J. Principios de Ética Biomédica. Barcelona: Masson S.A.; 1999.

13. Lantos JD. The Tragic Case of Charlie Gard. JAMA Pediatr 2017; 171(10): 935-936.

14. Gómez-Lobo A. Eutanasia y bienes humanos. Una contribución al debate actual. Texto inédito de conferencia dictada en Salón de honor, Casa Central, PUC; 2008.

15. Twycross R. Medicina paliativa: filosofía y consideraciones éticas. Acta Bioethica 2000; 6(1): 29-46.

16. Taboada P. Principios éticos relevantes en medicina paliativa. En: Organización Panamericana de la Salud, eds. Cuidados paliativos. Guía para el manejo clínico: 9-14.

17. Calipari M. El principio de lo éticamente adecuado en el uso de medios de preservación de la vida: entre el encarnizamiento terapéutico y el abandono del paciente. Vida ética 2008; 9(2): 163-174.

Recibido: 16 de febrero de 2019

Aceptado: 12 de marzo de 2019 